\title{
Development of Handkerchief Automatic Folding Device
}

\author{
Raiki Shigeta a*, Yuhki Kitazono a, \\ ${ }^{a}$ National Institute of Technology, Kitakyushu College, 5-20-1 Shii, Kokura Minami Ward, Kitakyushu City, Fukuoka \\ 802-0985, Japan \\ *Corresponding Author: k15081rs@apps.kct.ac.jp
}

\begin{abstract}
Housework is a daily job and a very hard job. Laundry is one of them. This paper is about the development of the handkerchief automatic folding device we developed. After removing the handkerchief from the washing machine, drying it and putting it on the device, our device will automatically fold it. We hope that this device will make everyday housework easier.
\end{abstract}

Keywords: Folding the Laundry, Folding System, Arduino, Handkerchief, Motor.

\section{Introduction}

$\mathrm{H}$ Housework such as cleaning, washing, shopping is indispensable to live a family life well, and it is hard work. Among them, in the case of a general household, the cleaning operation can be reduced by using a cleaning robot (Roomba, iRobot Japan G.K) or the like. In addition, as for shopping, agency and delivery services are provided by various companies. But what about laundry? Washing machines wash clothes, and if they have a drying function, they dry clothes. However, the washing machine does not take out clothes and hang them on a hanger or fold them for storage. A product (Folding Clothes Board, Daisaku Shoji Ltd.) that support folding clothes is on the market. In this product, clothes are placed on a board that bends in a specific direction, and then the board is folded to quickly fold the clothes. Although it is a convenient product full of ideas, it cannot be said that the burden of folding the laundry has been eliminated since the work of putting the clothes on the board and folding them is manual work. Furthermore, according to the "Assessment of housework activities, Reassessment with data in 2011, the lost profit (wage lost by giving up labor to the market) is over 88 trillion yen in 2011(1). Among them, those of laundry exceed 10 trillion yen. If you just do laundry, you can leave it to a washing machine. However, drying clothes that have been washed on a hanger or the like, removing them when they are dry, folding them, and storing them are manual work, which is a heavy burden on those who carry out the work. Besides, the burden is much heavier at the places where there are a large amount of laundry on a daily basis, such as welfare facilities, nursing homes or large families. Under such circumstances, a device for automatically folding various clothes has been developed, but at present, practical products for general household use have not been sold yet. A device for automatically folding clothes such as T-shirts has already been proposed in " Development of Robot to Fold T-shirt in the State of Hanging"(2).

Therefore, in this research, we propose a handkerchief automatic folding device and its mechanism to reduce the burden of laundry at home and welfare facilities as much as possible and aim to improve the quality of home life. This device can automatically fold a handkerchief by placing the handkerchief on the device. By using this device, "folding" can be automated among the five steps: washing, drying, removing from the hanger, folding and storing in the handkerchief laundry.

\section{Device Configuration}




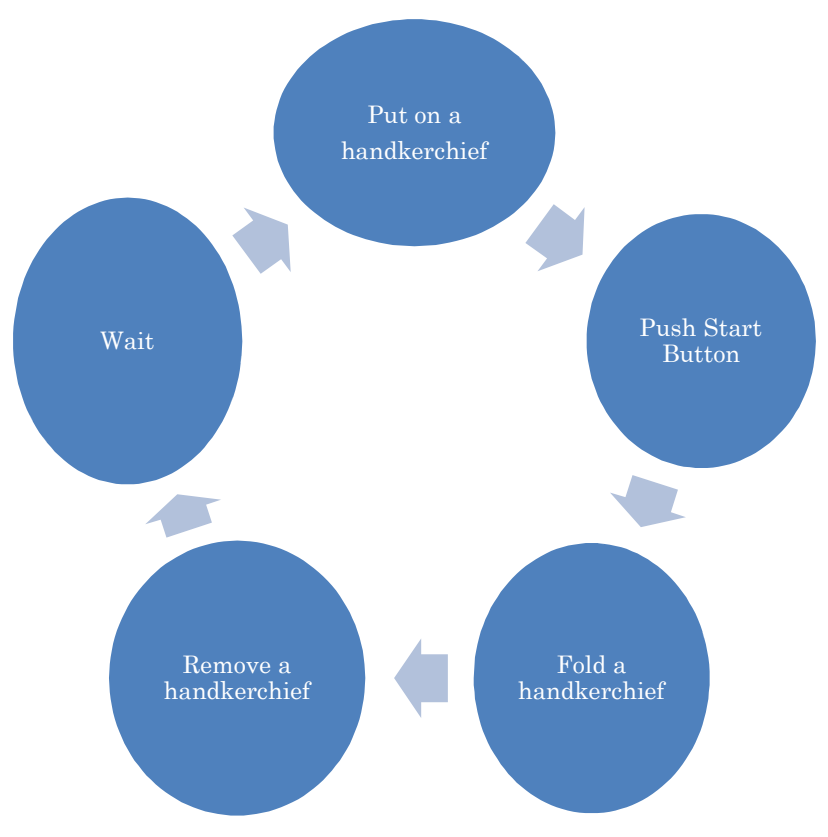

Fig. 1. Operation image

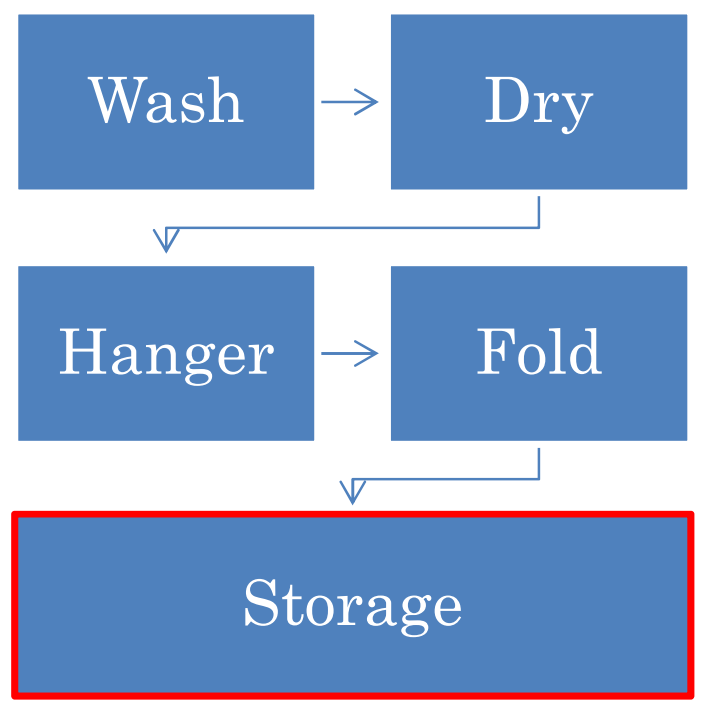

Fig. 2. Washing procedure

The operation image of the device is shown in Fig. 1, and the washing procedure is shown in Fig. 2. The area surrounded by a dotted line is an area where the device automatically performs. This device is constituted by a control unit by Arduino UNO and a drive unit by a servomotor. The user of this device first places a handkerchief on the device and presses the start button. When the start button is pressed, the handkerchief is folded in four by the installed servomotor. Finally, the user of the device removes the folded handkerchief from the device.

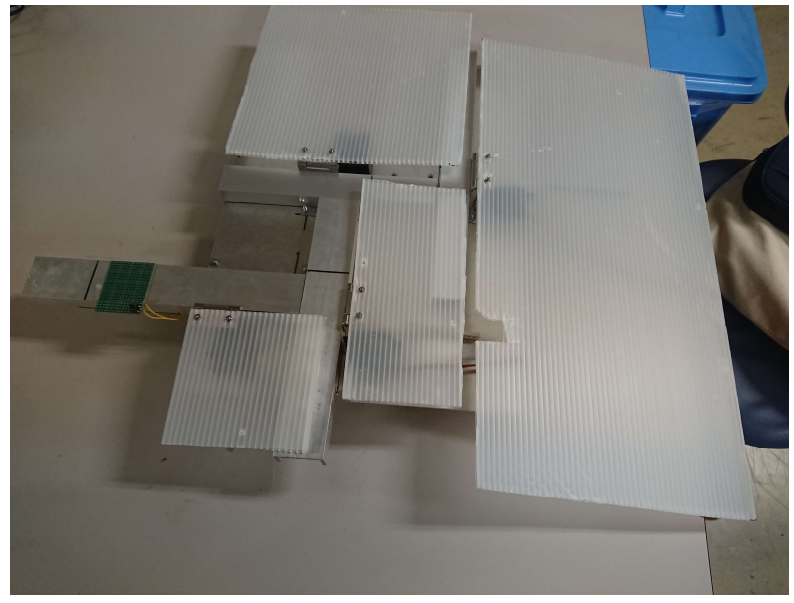

Fig. 3. Overview

The device waits until the user places the next handkerchief and presses the start button. As described above, the user places the handkerchief on the device and presses the start button. The device automatically folds the handkerchief and the user removes the folded handkerchief from the device. Automate the handkerchief's "folding" process is automated by repeating this series of operations.

\section{Prototype of Device}

Here, a prototype that implements the device described in Chapter 2 will be described. An overall view of the produced device is shown in FIG.3. The size of this device is $630 \mathrm{~W} \times 530 \mathrm{D} \times 60 \mathrm{H}(\mathrm{mm})$. The operation ofthe device starts when the user places the handkerchief in the center of the device and presses the start button. When the start button is pressed, the installed servomotor is driven to fold the handkerchief in four. This operation is realized using four servomotors and controlled by the Arduino UNO. Since this device was developed for general household use, AC $100 \mathrm{~V}$ is used as power supply, converted to $5 \mathrm{~V}$ and supplied to the device. The following shows the operation procedure and the operation image of this device.

\subsection{Handkerchief Installation}

The user of this device places the handkerchief in the center of the device. Thereafter, the device starts operation by the user pressing the start button located in the end of the device.

\subsection{Folding}




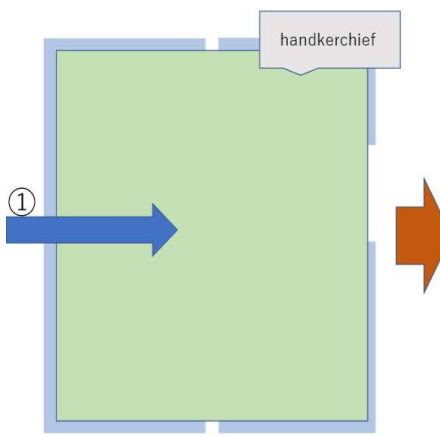

(a) Before folding
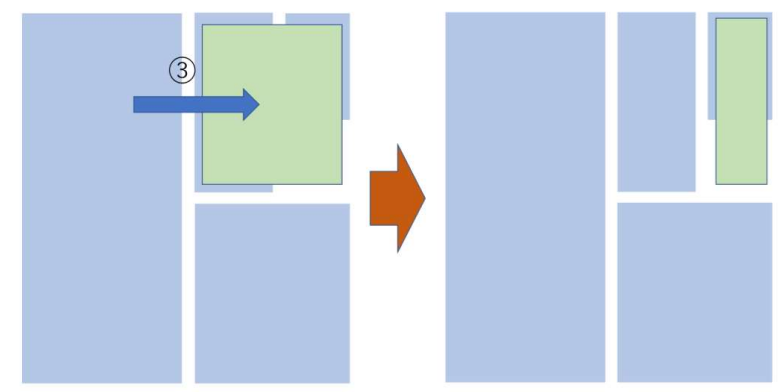

(c) Middle folding-2

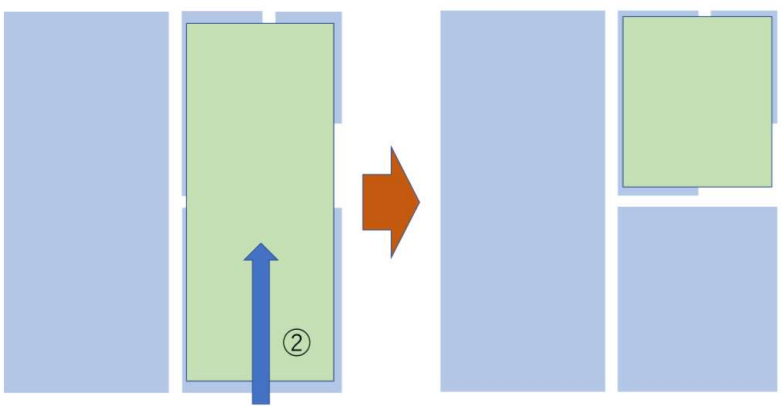

(b) Middle folding-1
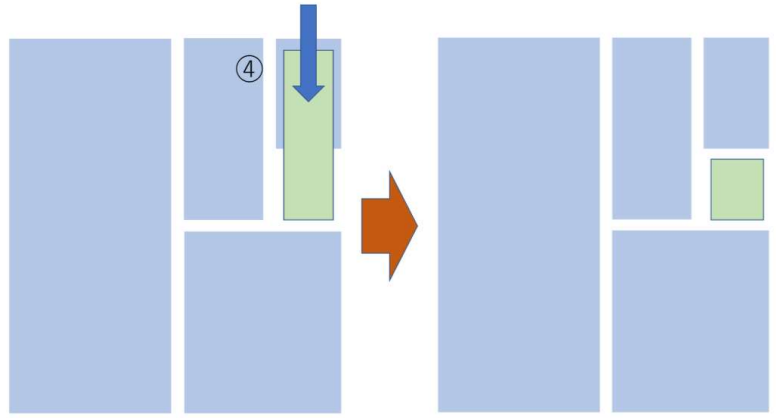

(d) After folding

Fig. 4. Operation image

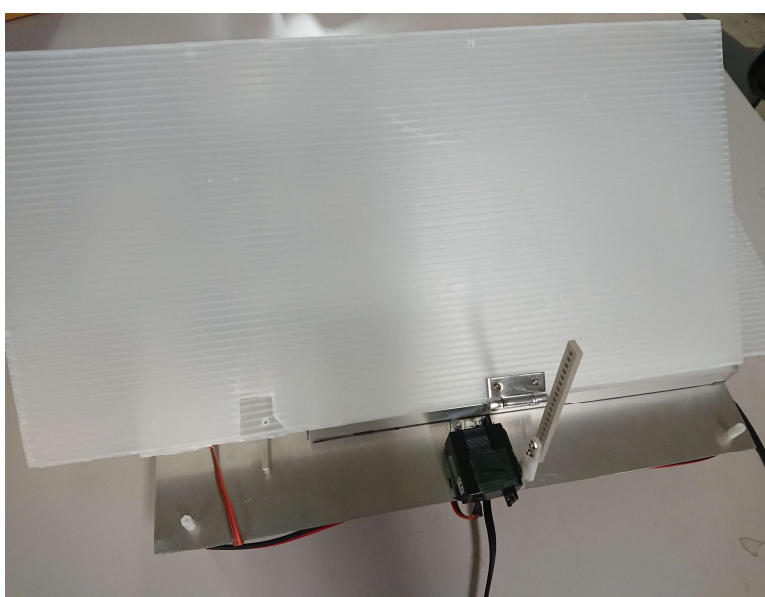

Fig. 5. Actuator

Using four servomotors, the plastic cardboard board is moved and the handkerchief into four is folded. Arduino UNO folds the handkerchief by rotating the servomotor $90^{\circ}$.

\subsection{Wait}

After folding the handkerchief, the device waits for the input of the next start button. The user of the device removes the folded handkerchief.

\section{Mechanism}

Each mechanism of this device is shown in 4.1 and 4.2

\subsection{Control Unit}

This device is controlled by Arduino UNO. The Arduino UNO controls the four servomotors and the start button that folds a handkerchief.

\subsection{Drive Part}

The mechanism of folding the handkerchief is shown in Fig. 5. By connecting the servomotor with the plastic cardboard board, the plate follows the drive of the servomotor. A handkerchief is folded by bending this board in each direction. 


\section{Experiment}

A handkerchief was folded using the handkerchief automatic folding device created in this study. In the experiment, a handkerchief of $100 \%$ cotton $470 \times 470$ $(\mathrm{mm})$ was used. Fig. 6 shows the operation of the device to fold the handkerchief.

\subsection{Equipment Performance}

It was measured whether one handkerchief could be

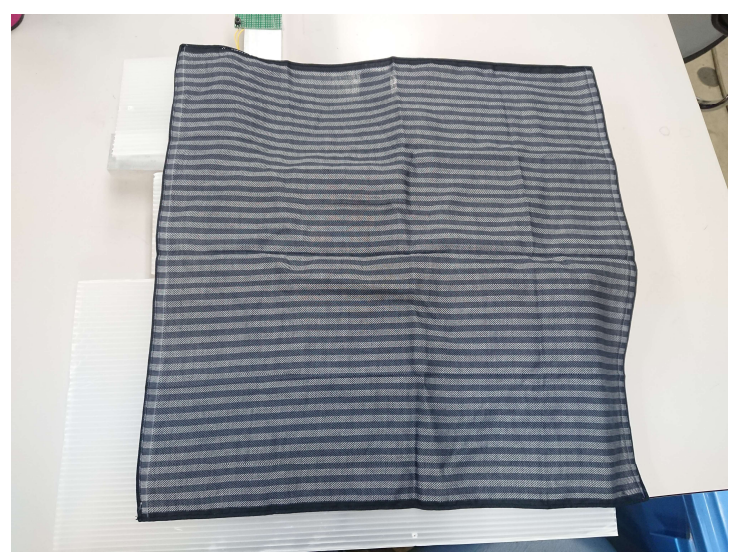

(a) Before folding

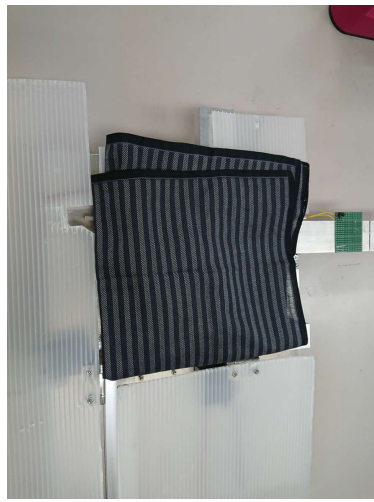

(b) Middle of folding-1

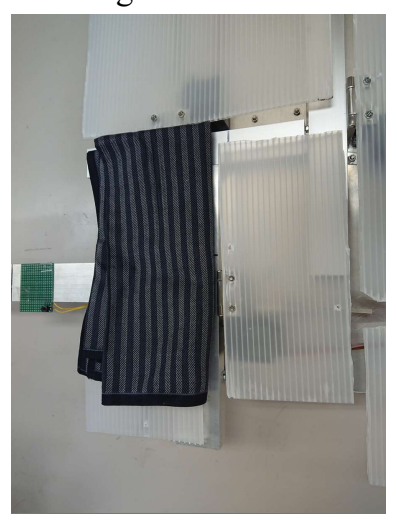

(c) Middle of folding-2

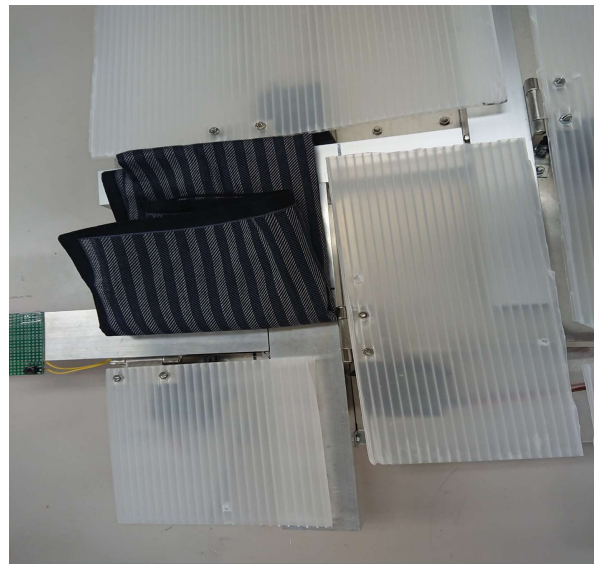

(d) After folding

Fig. 6. Folding a handkerchief folded and how long it took. In this experiment, after the handkerchief is placed on the device as shown in Fig. 6 (a), the device is operated until the handkerchief is completely folded as shown in Fig. 6 (d), and the time required is measured. As a result, it took about 20 seconds to fold one handkerchief. Assuming that there are three people in one household, you will have to wash around three handkerchiefs a day. By using this device, the user of the device can do other work for the one minute that it takes for the device to fold the three handkerchiefs. Therefore, it can be said that the burden of washing can be reduced by using this device.

\section{Conclusions}

In this study, we developed an automatic folding device for handkerchiefs. The developed device was able to automatically fold the handkerchief on the device. This can lead to reduce the burden of laundry at home and facilities. However, since this device cannot fold the handkerchief very well and does not operate unless the start button is pressed, it cannot be said that the burden of washing has been sufficiently reduced. In addition, since the noise generated during operation is large, from now on, we aim to implement the mechanism improvement that folds the handkerchief more beautifully, detects the handkerchief without pressing the start button, and automatically operates the device. Moreover, as a further function, the handkerchief is removed from the state of being hung on a hanger or the like, and we aim to implement a folding mechanism.

\section{References}

(1) Cabinet Office, Economic and Social Research Institute, Area and Specific account department, "About evaluation such as housework, Reassessment with 2011 data" (2013), https://www.mhlw.go.jp/toukei/saikin/hw/k-tyosa/k-tyo sa17/dl/10.pdf

(2) Ryunosuke Miyamoto, and Yuhki Kitazono : "Development of Robot to Fold T-shirt in the State of Hanging", The Japanese Journal of the Institute of Industrial Applications Engineers, Vol. 3, No. 1, pp. 2433 (Mar. 2015)

(3) Ministry of Health, Labor and Welfare, "Summary of 2017 national life basics investigation" (2018) 